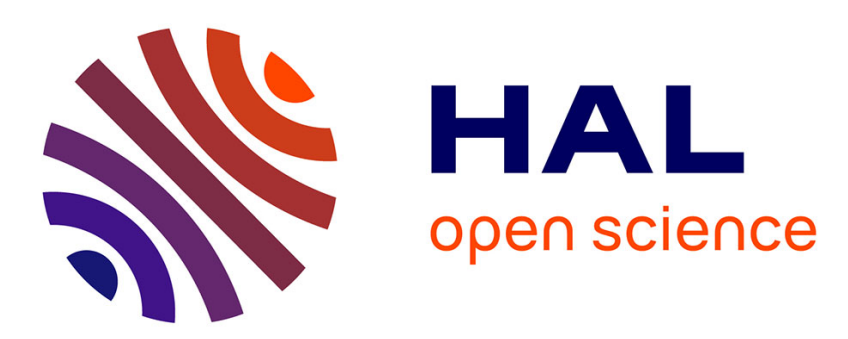

\title{
Numerical study of the short pre-arcing time in HBC fuses via an enthalpy formulation
}

\author{
Rachid Touzani, David Rochette, William Bussiere
}

\section{To cite this version:}

Rachid Touzani, David Rochette, William Bussiere. Numerical study of the short pre-arcing time in HBC fuses via an enthalpy formulation. Journal of Physics D: Applied Physics, 2007, 40, pp.4544-4551. 10.1088/0022-3727/40/15/026 . hal-00123743

\section{HAL Id: hal-00123743 \\ https://hal.science/hal-00123743}

Submitted on 10 Jan 2007

HAL is a multi-disciplinary open access archive for the deposit and dissemination of scientific research documents, whether they are published or not. The documents may come from teaching and research institutions in France or abroad, or from public or private research centers.
L'archive ouverte pluridisciplinaire HAL, est destinée au dépôt et à la diffusion de documents scientifiques de niveau recherche, publiés ou non, émanant des établissements d'enseignement et de recherche français ou étrangers, des laboratoires publics ou privés. 


\title{
Numerical study of the short pre-arcing time in $\mathrm{HBC}$ fuses via an enthalpy formulation
}

\author{
David Rochette ${ }^{1}$, Rachid Touzani ${ }^{2}$ and William Bussière ${ }^{1}$ \\ ${ }^{1}$ Laboratoire Arc Electrique et Plasmas thermiques, CNRS UMR 6069, Université \\ Blaise Pascal - IUT de Montluçon, Avenue Aristide Briand, BP 2235, 03101 \\ Montluçon cedex, France. \\ ${ }^{2}$ Laboratoire de Mathématiques, CNRS UMR 6620, Université Blaise Pascal \\ (Clermont-Ferrand II), Campus Universitaire des Cézeaux, 63177 Aubière cedex, \\ France. \\ E-mail: rochette@moniut.univ-bpclermont.fr, \\ rachid.touzani@univ-bpclermont.fr, william.bussiere@univ-bpclermont.fr
}

\begin{abstract}
In order to study the short pre-arcing time in HBC fuses, we use a mathematical model including phase change of the fuse-element heating coupled with the Laplace equation for the potential and the Ohm's law. The thermal model is based on the enthalpy formulation of the heat equation with a source term representing the Joule heating. For the time range considered (up to $10 \mathrm{~ms}$ ), we assume no heat transfer between the fuse-element and the surrounding sand. To solve numerically the governing equations, we employ a semi-implicit scheme for time integration and a finite element method for space discretization. Using electrical and thermal properties of the silver fuse-element, we present pre-arcing characteristics (temperature, current density, potential) for a fuse-element used in industrial protection circuits.
\end{abstract}




\section{Introduction}

High Breaking Capacity (HBC) fuse links are used since more than one century [1] in the current protection devices thanks to the fast interrupting of the highest fault currents and to the device basic simplicity. HBC fuses are generally composed of a single thin fuse-element strip or else a number of parallel-connected fuse-elements, usually in silver, with one or more shares of reduced cross section areas called notches [1]. These constrictions are designed with different shapes as circular, rectangular or trapezoidal. The conductor element is usually surrounded by silica sand and enclosed in a mechanical robust ceramic body.

Due to the fuse-element complex geometry and the filling material heterogeneities, the HBC fuses have a rather a complex behaviour. HBC fuses generally operate in two characteristic time ranges that involve different physical processes, named pre-arcing and arcing times. Let us briefly recall the physical processes which cause the fuse to limit and cut the fault currents [1,2]. During a fault current interruption, the current flow induces Joule heating of the fuse-element, especially around notches where the electrical resistivity is the highest due to cross section reduction. Once no balance is achieved between the electrical energy input and the dissipated heat energy, the temperature increases in the notch reaching the melting temperature of the constitutive metal and the fuse-element vaporizes. The time range starting when the current is large enough melt the fuse-element and ending with melting and vaporization is commonly known as the pre-arcing time. The fuse element notch disruption results then in the generation of an electric arc and energy is dissipated from the arc column towards the filling material. The electric arc induces a large voltage rise as well as an arc lengthening due to the fuse-element erosion (burn-back) leading to the arc extinction until the current reaches zero [2]. The time lapse between the initiation of the arc and the final arc extinction is known as the arcing time.

Pre-arcing times can be classified into three time ranges [3]:

(a) Short time (up to $10 \mathrm{~ms}$ ). For pre-arcing times up to approximately $1 \mathrm{~ms}$, the fuse-element temperature rise is almost adiabatic i.e. the heat loss by thermal diffusion between the notch and the remainder of the fuse element is negligible independently of the fuse-element material. For pre-arcing times between $1 \mathrm{~ms}$ and $10 \mathrm{~ms}$, thermal diffusion effects operate between the notch and the remainder of the fuse-element. They depend on the material properties. During these short times and taking into account the large ratio of thermal conductivities between the fuse-element $\left(k_{\mathrm{Ag}}(293 \mathrm{~K})=429.0 \mathrm{~W} \mathrm{~m}^{-1} \mathrm{~K}^{-1}\right)$ and the silica sand $\left(k_{\mathrm{SiO}_{2}}(293 \mathrm{~K})=0.3 \mathrm{~W} \mathrm{~m}^{-1} \mathrm{~K}^{-1}\right)$, there is no heat transfer from the fuse-element to the silica sand.

(b) Medium time (10 ms to $1 \mathrm{~s})$. For pre-arcing times between $10 \mathrm{~ms}$ and $1 \mathrm{~s}$, the heat transfer is carried out firstly from the notch to the remainder element and secondly to the surrounding sand depending on the sand morphometric properties. 
(c) Long time $(>1 \mathrm{~s})$. For pre-arcing times larger than $1 \mathrm{~s}$, the heating process is conducted away through the element to the fuse end caps and connecting cables and also through the sand filler. In addition, the pre-arcing characteristics in this time range are affected by the M-effect [4, 3] when alloy is added through the constitutive element material.

In the last decades, numerous numerical investigations have studied the pre-arcing phase in HBC fuses using the finite difference method [5, 6, 7, 8, 9] or the finite element method $[10,11,12,13,14,15,16]$. Another approach is developed in [17, 18] where the temperature distribution as well as thermal and electrical resistances of the different parts of the fuse are described by exact or semi-empirical equations. Other works depict the fuse as an equivalent thermal resistance-capacitance network $[3,19]$ in order to evaluate the temperature distribution in the fuse-element. These earlier works have allowed to obtain, for a particular fuse-element, the inverse prearcing time/current characteristics. The numerical tools allow to develop new fuse designs without achieving a laborious iterative experimental process. Moreover, many parameters such as current flow lines, temperature distribution can be determined for various fuse-element geometries and different materials. The shape of notches can also be designed with the help of such calculations. In all quoted works, the fuse-element heating is calculated until the melting temperature is reached. Consequently the prearcing time does not include the time range that is necessary to pass the liquid phase to the vapour phase because no phase transition is included in these fuse models.

In this paper, we limit our study for the first time range (up to $10 \mathrm{~ms}$ ) where heat transfer between the fuse-element and the filling sand is not significant and the power dissipated by thermal radiation is neglected. Consequently a two-dimensional geometry is used to study the pre-arcing period for industrial fuse-elements. We propose a model and a specific numerical method taking into account phase transition of the heated fuse-element, allowing to extend the simulation until the vaporization temperature is reached. This complete approach is new up to our knowledge. It is motivated by the fact that our final goal is to obtain a complete model of the fuse operation in which the present model will include fluid flow [20, 21] taking into account thermodynamical properties of $\mathrm{AgSiO}_{2}$ plasma [22, 23].

The paper is organized as follows: in section 2, we present the mathematical model of the fuse-element heating. In section 3, we describe the characteristics of the fuse-element considered and section 4 is devoted to the numerical method. In section 5 , we present the numerical results of the pre-arcing time characteristics.

\section{Model of the fuse-element heating}

To model the pre-arcing regime in a HBC fuse in the case of short time periods, we assume no heat transfer between the fuse-element and the filling material. We also use a two-dimensional model coupling the electrical and the thermal problems including solid - liquid phase change of the fuse-element material. The nonlinear transient heat 
conduction equation is written in terms of the enthalpy function. The electric part is modelled by the Laplace equation governing a quasi-static electric potential where induction effects are neglected. Assuming the Ohm's law, the governing equations of the pre-arcing period are described by the set of equations:

$$
\left\{\begin{array}{l}
\frac{\partial H}{\partial t}-\nabla \cdot(k \nabla T)=\frac{|\mathbf{J}|^{2}}{\sigma} \\
\nabla \cdot(\sigma \nabla V)=0 \\
\mathbf{E}=-\nabla V \\
\mathbf{J}=\sigma \mathbf{E}
\end{array}\right.
$$

where $T$ is the temperature field, $H=H(T)$ is the volumetric enthalpy, $k=k(T)$ and $\sigma=\sigma(T)$ are respectively the thermal and electrical conductivities depending on the temperature, $\mathbf{J}$ is the vector of current density, $\mathbf{E}$ is the electric field vector and $V$ is the electric potential.

The enthalpy function $H$ is defined as a primitive of the heat capacity with respect to temperature, i.e.

$$
H(T)=\int_{T_{\mathrm{ref}}}^{T} \rho c(s) d s,
$$

where $\rho$ is the density, $c$ is the specific heat capacity at constant pressure which depends on the temperature and $T_{\text {ref }}$ is a reference temperature. For the case of isothermal phase change (valid for a pure material), the enthalpy function has a jump discontinuity at the melting temperature $T_{m}$ and the function may be written as:

$$
H(T)= \begin{cases}\int_{T_{\mathrm{ref}}}^{T} \rho c_{s}(s) d s & \text { if } T<T_{m} \\ \int_{T_{\mathrm{ref}}}^{T_{m}} \rho c_{s}(s) d s+\rho L+\int_{T_{m}}^{T} \rho c_{l}(s) d s & \text { if } T \geq T_{m}\end{cases}
$$

where $c_{s}$ and $c_{l}$ are respectively the specific heat capacities at constant pressure of the solid and liquid phases and $L$ denotes the latent heat. To solve the transient electric-thermal problem, equations (1) must be supplemented by boundary conditions as described in section 3 .

\section{Fuse-element description}

Our aim is to study the presented model through a sample of fuse-element geometry used in industrial devices. The sample is assumed to have a projection on the plane $(O x y)$, given in figure 1 , and whose thickness, in the $z$-direction, is $e$. 


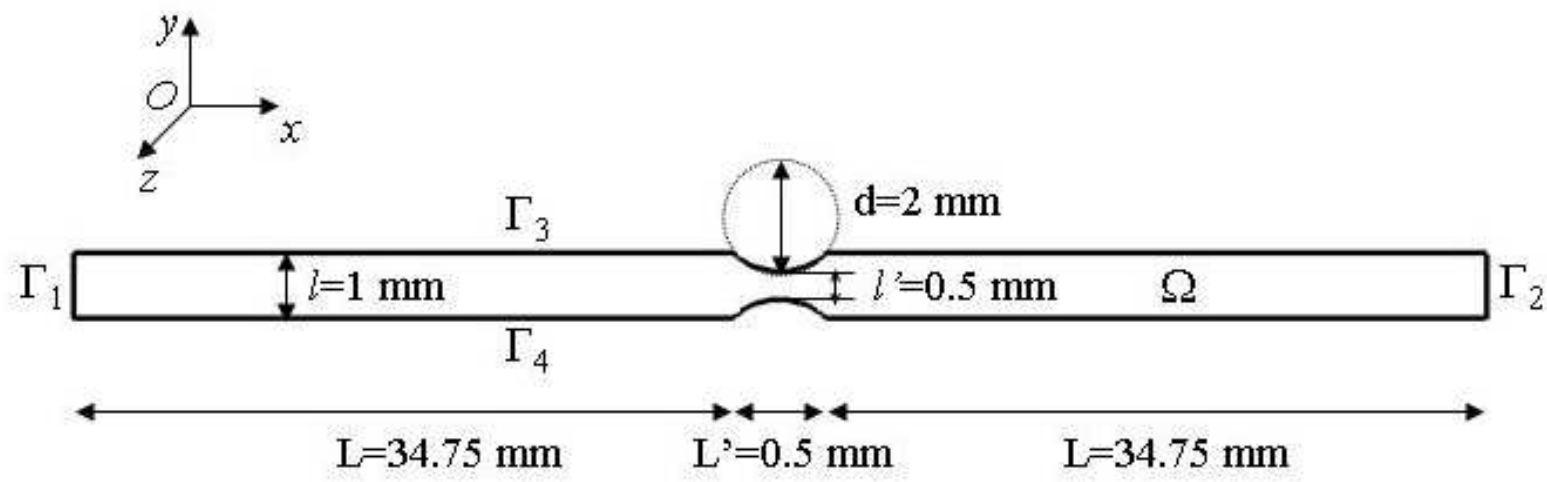

Figure 1. Two-dimensional fuse-element geometry.

The fuse-element is made of pure silver for simulations (experimental fuse-elements are generally made of $99.99 \%$ pure silver), the fuse-element is provided with only one notch and its dimensions in plane $(O x y)$ are outlined in figure 1 and following the $z$-direction its thickness is $e=0.105 \mathrm{~mm}$.

As far as boundary conditions are concerned, we impose the following ones:

(i) On boundary $\Gamma_{1}$, we impose for the electric problem a Neumann condition representing the current density flux through the fuse cross section and for the thermal problem, we impose a homogeneous Neumann condition expressing a thermal insulation (contact resistance with electrode is assumed ideal):

$$
\begin{gathered}
\sigma(T) \frac{\partial V}{\partial \mathbf{n}}=\frac{I(t)}{\left|\Gamma_{1}\right|}, \\
k(T) \frac{\partial T}{\partial \mathbf{n}}=0,
\end{gathered}
$$

where $\mathbf{n}$ is the outward unit normal to the boundary, $I(t)$ is the total current in the fuse-element and $\left|\Gamma_{1}\right|=l e$ is the cross section area associated to $\Gamma_{1}$.

(ii) On boundary $\Gamma_{2}$, a Dirichlet condition is prescribed for the reference potential and a homogeneous Neumann condition for the temperature:

$$
\begin{aligned}
& V=0, \\
& k(T) \frac{\partial T}{\partial \mathbf{n}}=0 .
\end{aligned}
$$

(iii) On boundaries $\Gamma_{3}$ and $\Gamma_{4}$, we impose a homogeneous Neumann condition assuming that heat transfer between the fuse-element and the surrounding sand is not significant for the considered currents:

$$
\begin{aligned}
& \sigma(T) \frac{\partial V}{\partial \mathbf{n}}=0, \\
& k(T) \frac{\partial T}{\partial \mathbf{n}}=0 .
\end{aligned}
$$


To complete the model, we add a constitutive function that describes the enthalpy versus temperature for the considered material. For a silver fuse-element, the relation between enthalpy and temperature [24], if constant specific heat and density are assumed for each phase and considering the situation of an isothermal phase change, is given by (see figure 2(a))

$$
H(T)= \begin{cases}\rho c_{s} T+H_{\mathrm{ref}} & \text { if } T<T_{m}, \\ \rho c_{s} T_{m}+H_{\mathrm{ref}}+\rho L+\rho c_{l}\left(T-T_{m}\right) & \text { if } T \geq T_{m}\end{cases}
$$

In view of the numerical treatment described in section 4 , the reciprocal form $\beta(H)$ of $H(T)$ is given by (see figure $2(\mathrm{~b})$

$$
\beta(H)= \begin{cases}\frac{H-H_{\mathrm{ref}}}{\rho c_{s}} & \text { if } H<H_{s}=\rho c_{s} T_{m}, \\ T_{m} & \text { if } H_{s} \leq H<H_{s}+\rho L, \\ T_{m}+\frac{H-\rho c_{s} T_{m}-H_{\mathrm{ref}}-\rho L}{\rho c_{l}} & \text { if } H \geq H_{s}+\rho L .\end{cases}
$$
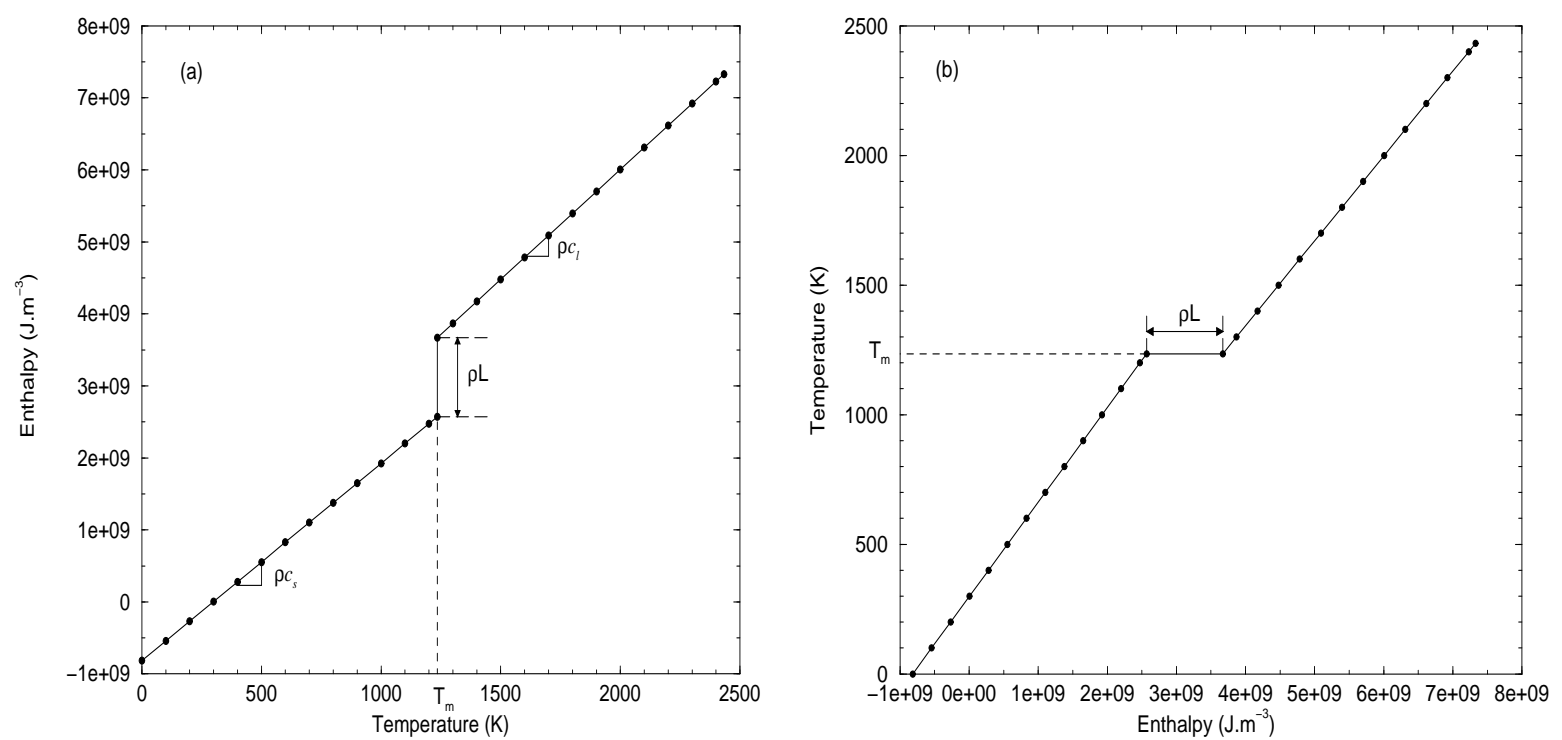

Figure 2. Enthalpy versus temperature $H(T)$ relation (a) and temperature versus enthalpy $\beta(H)$ relation (b) for silver material.

The material characteristics used for the simulation are enumerated in table 1. Moreover, we consider a thermal conductivity function $k(T)$ (see [25]) and an electric conductivity function $\sigma(T)$ (see [26]) dependent on temperature where the thermal and electric conductivity jumps for the phase change are respectively:

$$
\begin{aligned}
& \Delta k\left(T_{m}\right)_{s-l}=k_{s}\left(T_{m}\right)-k_{l}\left(T_{m}\right)=177.92 \mathrm{~W} \mathrm{~m}^{-1} \mathrm{~K}^{-1} \\
& \Delta \sigma\left(T_{m}\right)_{s-l}=\sigma_{s}\left(T_{m}\right)-\sigma_{l}\left(T_{m}\right)=6.1037 \times 10^{6} \mathrm{~S} \mathrm{~m}^{-1} .
\end{aligned}
$$


Table 1. Physical properties of the silver.

\begin{tabular}{ll}
\hline Silver density & $\rho=10490 \mathrm{~kg} \mathrm{~m}^{-3}$ \\
Solid heat capacity & $c_{s}=261.36 \mathrm{~J} \mathrm{~kg}^{-1} \mathrm{~K}^{-1}$ \\
Liquid heat capacity & $c_{l}=310.4 \mathrm{~J} \mathrm{~kg}^{-1} \mathrm{~K}^{-1}$ \\
Melting temperature & $T_{m}=1235 \mathrm{~K}$ \\
Boiling temperature & $T_{v}=2433 \mathrm{~K}$ \\
Latent heat & $L=1.05 \times 10^{5} \mathrm{~J} \mathrm{~kg}^{-1}$ \\
Reference enthalpy & $H_{\text {ref }}=-8.17 \times 10^{8} \mathrm{~J} \mathrm{~m}^{-3}$ \\
Thermal conductivity & $k_{s}=429 \mathrm{~W} \mathrm{~m} \mathrm{~K}^{-1}$ at $293 \mathrm{~K}$ \\
Electric conductivity & $\sigma_{s}=64.17 \times 10^{6} \mathrm{~S} \mathrm{~m}^{-1}$ at $293 \mathrm{~K}$ \\
\hline
\end{tabular}

\section{Numerical method}

We start by presenting the time integration scheme and then consider space discretization by a finite element method.

\subsection{Time discretization}

To integrate in time the resulting system, we use the semi-implicit scheme described in $[27,28]$ where the time discretization is done by the so called Chernoff scheme. This one consists in a relaxation of the relation between the temperature and the enthalpy. Let $\Delta t$ denote the time step and let for a function $f(t), f^{n}$ stands for the time approximation of $f(n \Delta t)$. Given $H^{n}, \mathbf{J}^{n}, k^{n}=k\left(\beta\left(H^{n}\right)\right)$ and $\sigma^{n}=\sigma\left(\beta\left(H^{n}\right)\right)$, we define the time integration scheme by:

$$
\begin{aligned}
& \nabla \cdot\left(\sigma^{n} \nabla V^{n}\right)=0, \\
& \mathbf{E}^{n}=-\nabla V^{n}, \\
& \mathbf{J}^{n}=\sigma^{n} \mathbf{E}^{n}, \\
& \gamma \frac{T^{n+1}-\beta\left(H^{n}\right)}{\Delta t}-\nabla \cdot\left(k^{n} \nabla T^{n+1}\right)=\frac{\left|\mathbf{J}^{n}\right|^{2}}{\sigma^{n}}, \\
& H^{n+1}=H^{n}+\gamma\left(T^{n+1}-\beta\left(H^{n}\right)\right),
\end{aligned}
$$

with boundary conditions described as in section 3 .

Here above, $\gamma$ is a relaxation parameter assumed to satisfy the condition

$$
0<\gamma \leq \frac{1}{\max \beta^{\prime}(H)}
$$

for stability requirements (see $[27,28]$ ) where $\beta^{\prime}(H)$ is the derivative of the function $H \mapsto \beta(H)$. It is noteworthy that $T^{n}$ is a pseudo-temperature at $t=n \Delta t$, the actual temperature field being $\beta\left(H^{n+1}\right)$. 


\subsection{Space discretization and variational formulation}

In order to allow for various fuse-element geometries, we use a simple $P_{1}$ finite element method to discretize in space the system (5)-(9). For this, we shall resort to a variational formulation. Let $\phi=\phi(x, y)$ stand for an arbitrary regular function and multiplying equation (8) by $\phi$ and integrating on $\Omega$, we obtain

$$
\frac{\gamma}{\Delta t} \int_{\Omega} T^{n+1} \phi d \Omega-\int_{\Omega} \nabla \cdot\left(k^{n} \nabla T^{n+1}\right) \phi d \Omega=\frac{\gamma}{\Delta t} \int_{\Omega} \beta\left(H^{n}\right) \phi d \Omega+\int_{\Omega} \frac{\left|\mathbf{J}^{n}\right|^{2}}{\sigma^{n}} \phi d \Omega .
$$

Using the Green formula and taking into account the Neumann boundary conditions (2)-(4), we deduce

$$
\frac{\gamma}{\Delta t} \int_{\Omega} T^{n+1} \phi d \Omega+\int_{\Omega} k^{n} \nabla T^{n+1} \cdot \nabla \phi d \Omega=\frac{\gamma}{\Delta t} \int_{\Omega} \beta\left(H^{n}\right) \phi d \Omega+\int_{\Omega} \frac{\left|\mathbf{J}^{n}\right|^{2}}{\sigma^{n}} \phi d \Omega .
$$

Similarly, we multiply equation (5) by a regular test function $\psi=\psi(x, y)$ that satisfies

$$
\psi=0 \text { on } \Gamma_{2},
$$

and integrate over $\Omega$ to obtain

$$
\int_{\Omega} \sigma^{n} \nabla V^{n} \cdot \nabla \psi d \Omega=\frac{I(t)}{\left|\Gamma_{1}\right|} \int_{\Gamma_{1}} \psi d \Gamma_{1}
$$

After triangulating the domain $\Omega$, the unknown functions $T, H, V, \mathbf{E}$ and $\mathbf{J}$ are approximated by piecewise linear functions and this results in a linear system of equations for each time step $\Delta t$.

\section{Numerical results and discussion}

As described in section 3, we impose a current $I(t)$ similar to an operating a.c. current supplied by a single-phase $100 \mathrm{kVA}$ transformer (see [29] for more details). During the pre-arcing time, the current is described by the classical relation

$$
I(t)=\frac{V_{M}}{\sqrt{R^{2}+(L \omega)^{2}}}\left(\sin (\omega t+\theta-\varphi)-\sin (\theta-\varphi) e^{-\frac{R}{L} t}\right),
$$

where $R$ and $L$ are respectively the total resistance and the total inductance of the circuit, $\omega$ is the supplied angular frequency, $V_{M}$ is the maximum voltage of the power supply, $\theta$ is the circuit closing angle and $\varphi$ denotes the power factor angle of the circuit. All the computations have been performed using the OFELI library (see [30]) and the calculations are stopped when the boiling temperature is reached indicating the end of the total pre-arcing time. 
The fuse-element, described in figure 1, is discretized with an unstructured mesh of 8917 triangles and 4958 nodes where the notch area is meshed more finely. At the initial time, the fuse-element temperature is fixed at $T_{0}=300 \mathrm{~K}$ and we have imposed a constant time step $\Delta t=10^{-5} \mathrm{~s}$. The total pre-arcing time is obtained after $5.35 \mathrm{~ms}$ time simulation which corresponds to the period preceding vaporization of the fuse-element and the initiation of an electric arc.

Figure 3 shows isothermal contours in the fuse-element close to the notch obtained from the simulation at $t=5.35 \mathrm{~ms}$. The temperature distribution is almost homogeneous in the fuse-element strip around $500 \mathrm{~K}$. As expected, the Joule effect is more important as one gets close to the notch involving a temperature rise. The hottest points are located on the notch boundaries and the vaporization will take place in these points and will produce a gap generating an electric arc.

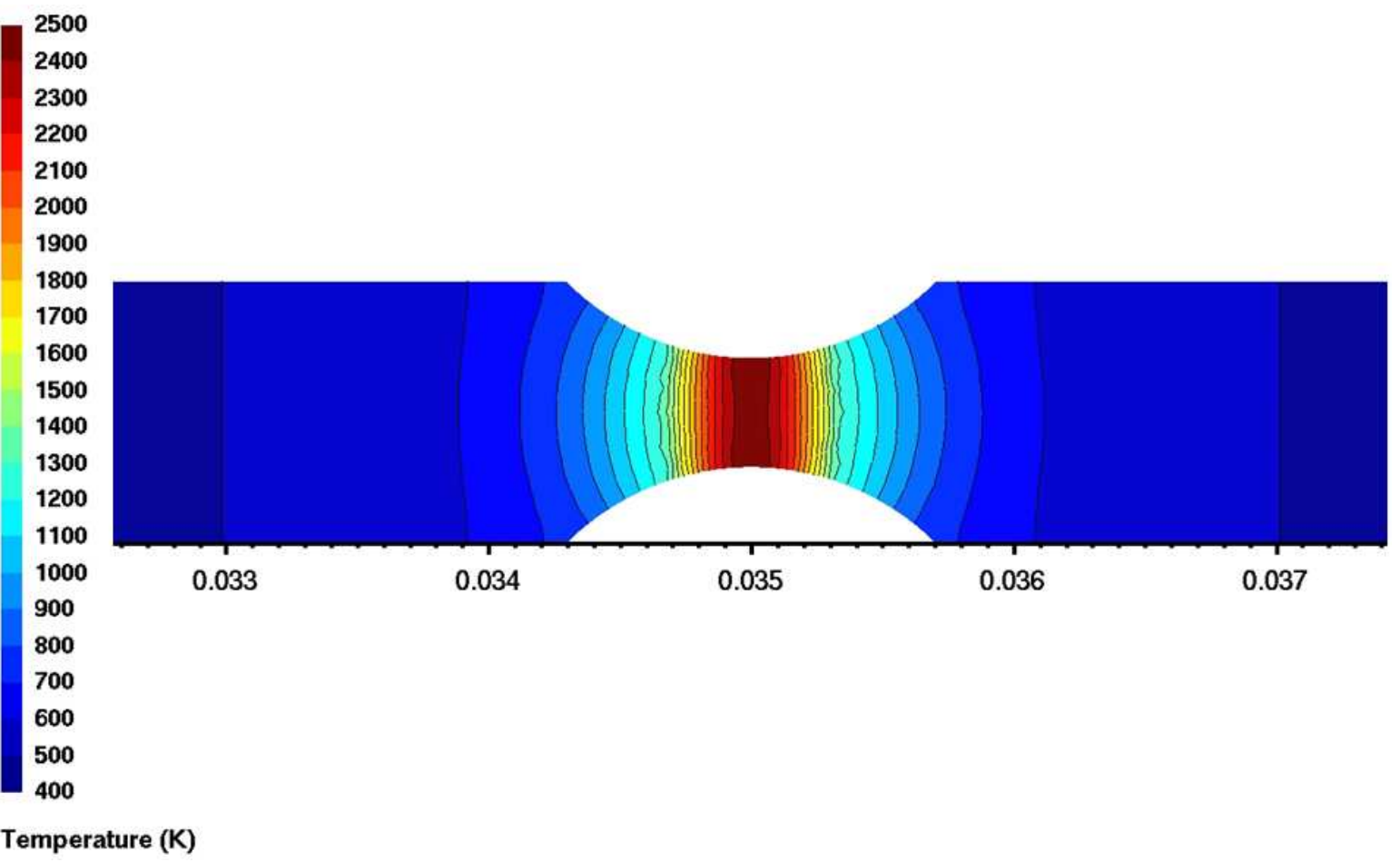

Figure 3. Temperature distribution in the fuse-element at time $t=5.35 \mathrm{~ms}$.

Figure 4 represents the temperature and the current density norm distributions at time $t=5.35 \mathrm{~ms}$ in function of the fuse-element length $x$ at $y$-coordinate $0.5 \mathrm{~mm}$ (which corresponds to the half height of the fuse-element). The current density varies along the length of the fuse-element and the current density vector is not axial close to the notch due to the particular shape of the restricted section. Clearly, the highest current densities occur in the restricted section inducing the highest temperature. Within the fuse-element strip, some important temperature gradients take place close to the notch. 

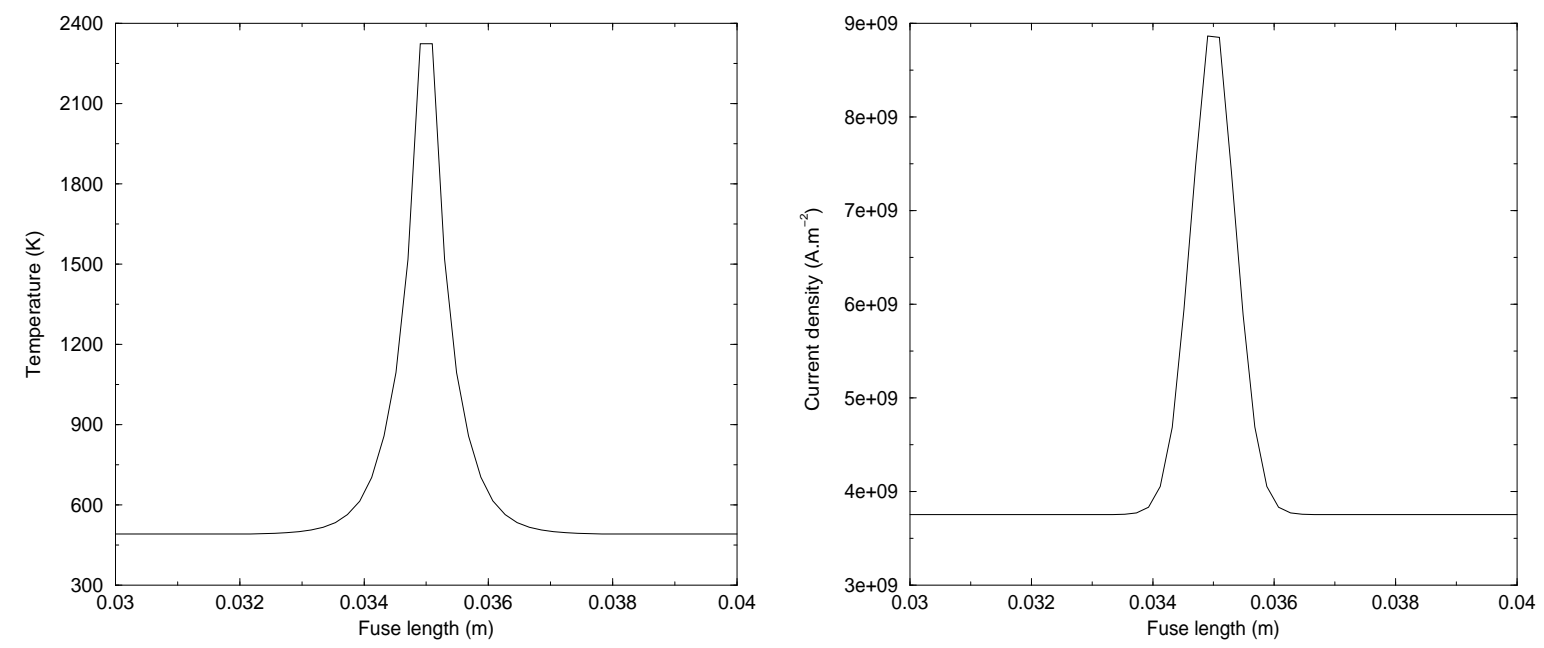

Figure 4. Temperature and current density norm distributions in the fuse-element at time $t=5.35 \mathrm{~ms}$ following the $x$-direction at $y=0.5 \mathrm{~mm}$.

Figure 5 gives the potential distribution in the fuse-element for four time values in function of length $x$ at $y$-coordinate $0.5 \mathrm{~mm}$. The voltage drop along the fuse-element as well as the voltage drop along the strangled constriction increase during the pre-arcing time due to the rise of the notch and fuse-element resistances induced by the decrease of the electric conductivity when the temperature increases.

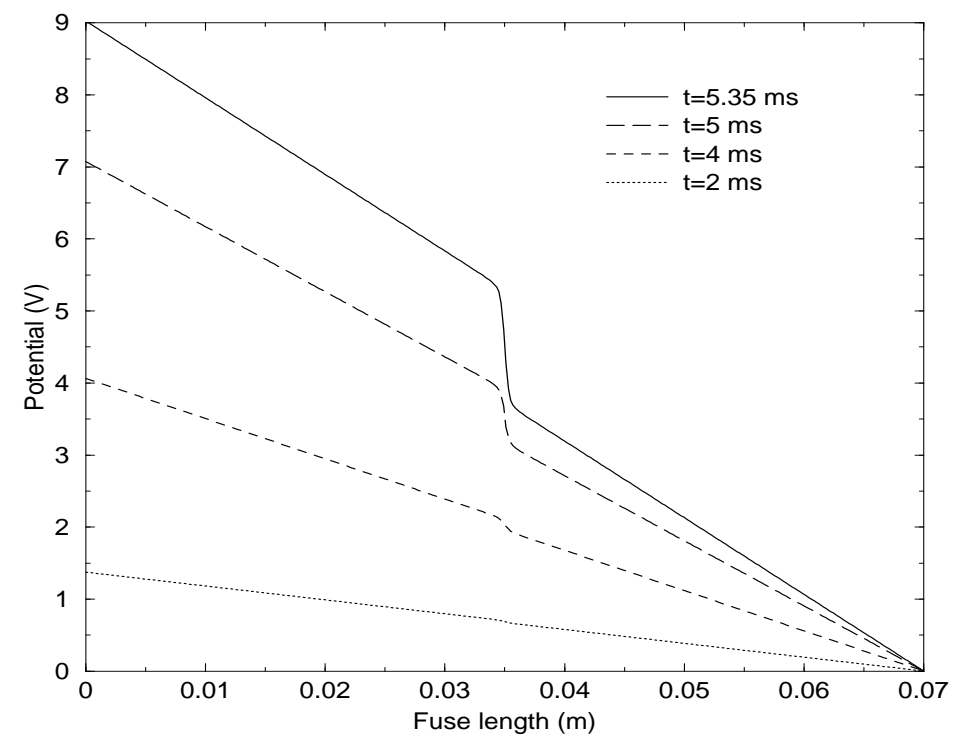

Figure 5. Potential distribution in the fuse-element at times $t=2 \mathrm{~ms}, t=4 \mathrm{~ms}$, $t=5 \mathrm{~ms}$ and $t=5.35 \mathrm{~ms}$ following the $x$-direction at $y=0.5 \mathrm{~mm}$.

The temperature history in the notch center of coordinates $(35 \mathrm{~mm}, 0.5 \mathrm{~mm})$ during the pre-arcing period is given in figure 6 . The power losses increase considerably after melting, around $t=5.05 \mathrm{~ms}$, due to the higher resistance of the fuse-element conducting 
path. Indeed, the electric conductivity drops strongly when solid - liquid transition occurs $\left(\Delta \sigma\left(T_{m}\right)_{s-l}=\sigma_{s}\left(T_{m}\right)-\sigma_{l}\left(T_{m}\right)=6.1037 \times 10^{6} \mathrm{~S} . \mathrm{m}^{-1}\right)$. As a result, the temperature profile during the total pre-arcing time can be divided in three steps. First, the time range necessary to reach the melting point which represents $92.5 \%$ of its final value. Second, the short time interval, included between $t=4.95 \mathrm{~ms}$ and $t=5.05 \mathrm{~ms}$, where the fuse-element temperature is roughly a constant equal to the melting temperature. This time range represents $1.9 \%$ of the total pre-arcing time and corresponds to the necessary time to supply the latent heat energy. Third, the subsequent period representing $5.6 \%$ of the pre-arcing time, going from the melting temperature to the boiling temperature of the silver fuse-element. The first time range is much longer in comparison with the two others and consequently these latter times do not significantly affect the overall pre-arcing time. For this reason, earlier works (for example [5, 15]) do not take them into account. Note that in the model, we assume that the liquid material does not flow away from its original position which may lead to errors because the current path is no longer well defined [1].

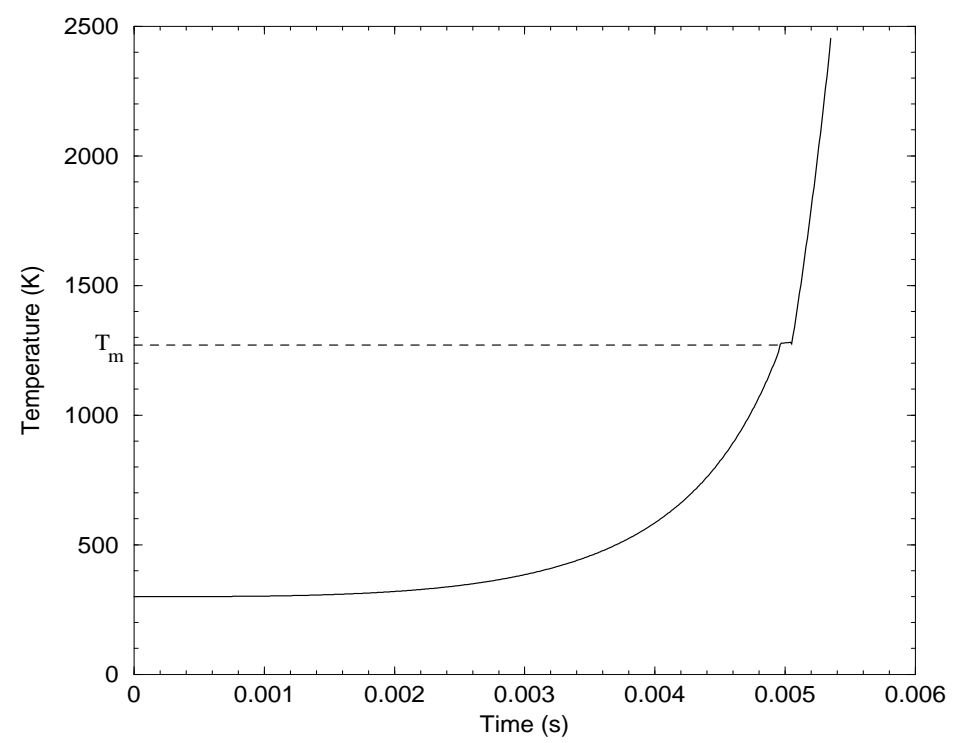

Figure 6. Temperature evolution in the notch center during the total pre-arcing period.

In figure 7, we compare time evolution of the voltage drop during the pre-arcing period obtained respectively by simulation and experiment. The experimental data, plotted during pre-arcing and arcing times, have been measured during a fuse operation test. The simulated current, which is a source data for the model, is obtained with equation (10) where circuit characteristics are referenced in [29]. In order to compare simulation and experiment with the same Joule integral $\int_{0}^{t_{f}} I^{2}(t) d t$, we have fitted the experimental parameters such as resistance and inductance of the test circuit to obtain the same current. We obtain for the simulated voltage drop a good agreement with the experimental voltage and the pre-arcing times differ of $0.25 \mathrm{~ms}$. This time deviation 
is in a large measure due to the geometrical shape difference of the notch between the simulated and the tested fuse-element. Indeed, as shown in figure 8, the experimental fuse-element is not perfectly symmetrical on the level of the notch and the notch width is slightly lower than $0.5 \mathrm{~mm}$. Consequently, the lower cross section area induces a more important Joule effect and a lower pre-arcing time.

Experimentally, we note at $t=5.1 \mathrm{~ms}$ a period of fast increase of voltage drop which is attributed to the transition liquid - vapor of the material, the electric arc is generated and is characterized by a ionized silver plasma of higher resistance.
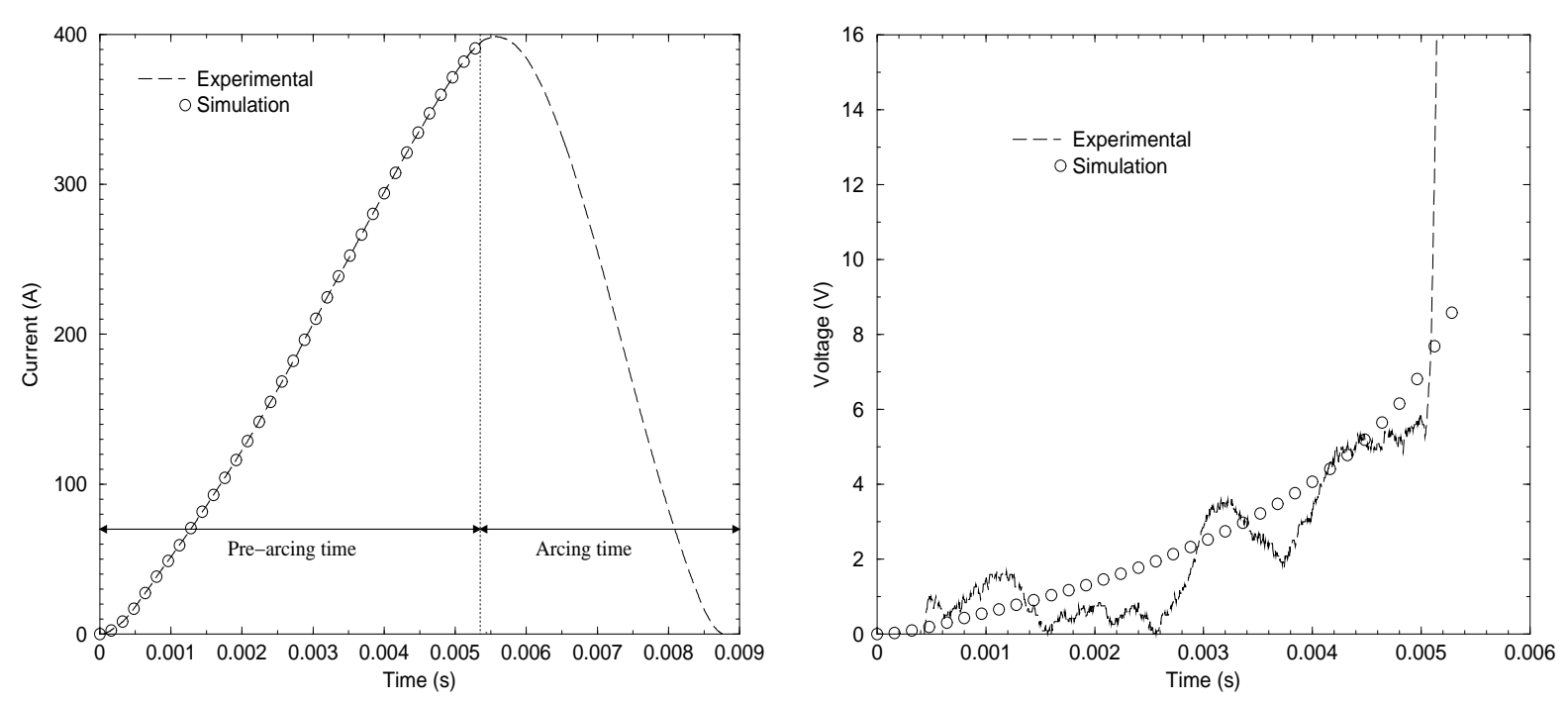

Figure 7. Current and voltage obtained by simulation (symbol o) and recording of a short-circuit experimental test (dashed line).

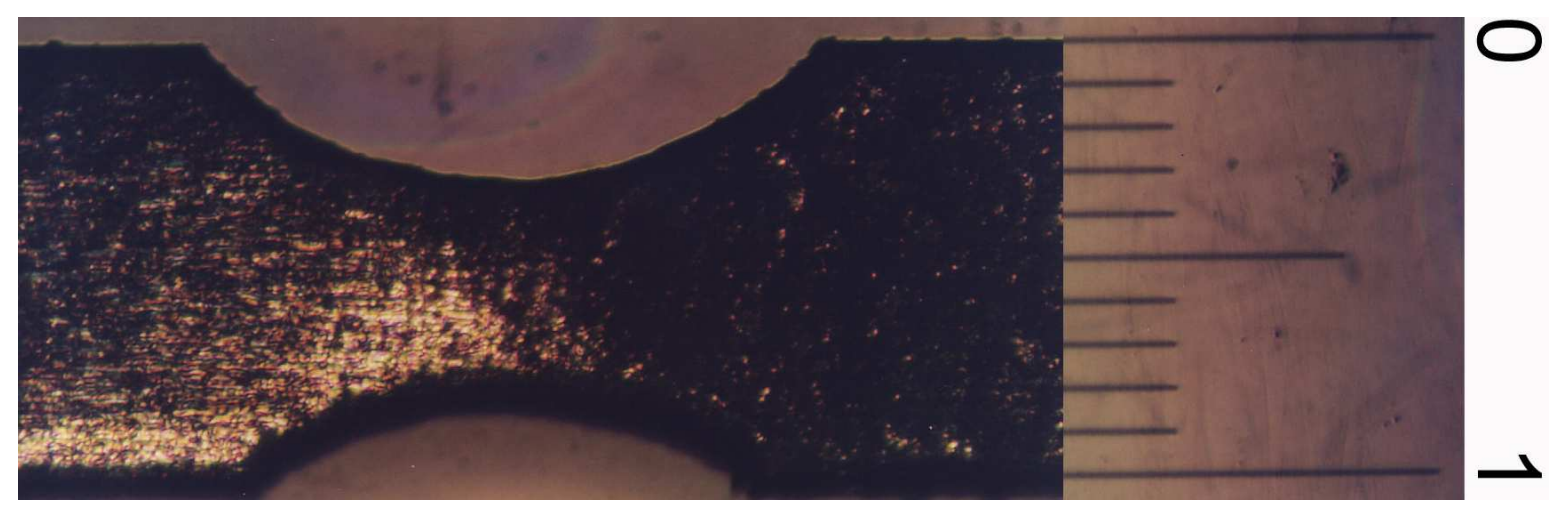

Figure 8. Microscopic picture of the experimental silver fuse-element geometry (the scale unity is in millimeter). 
The fuse resistance evolution during the pre-arcing period given in figure 9 is obtained by the relation $R_{\text {fuse }}=\Delta V / I(t)$ where $\Delta V$ denotes the voltage drop along the fuseelement. When the melting point is reached, the fuse resistance increases rapidly due to the higher resistivity of the liquid phase compared to the solid phase.

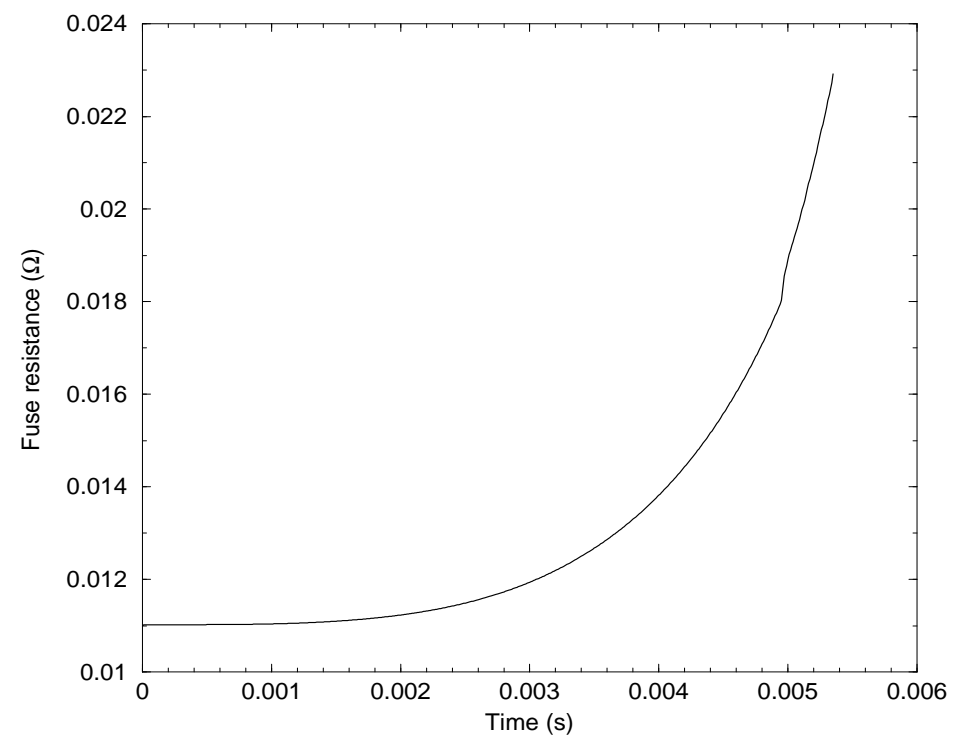

Figure 9. Fuse resistance evolution during the pre-arcing period.

\section{Conclusion}

We have presented a two-dimensional mathematical model for the short pre-arcing period in HBC fuses assuming no heat transfer between the fuse-element and the surrounding silica sand. The enthalpy formulation of the thermal problem allows to take into account the phase transitions of the fuse-element and consequently to extend the simulation until the boiling point is reached. A specific numerical scheme for the heat equation with phase transition is employed to obtain an approximate solution of the temperature. Simulation of the pre-arcing time for a fuse-element in pure silver with circular notch has been realized. As expected the Joule effect induces a temperature rise close to the notch involving the melting and the vaporization of the fuse-element. Imposing a total current similar to an operating a.c. current supplied by a transformer allows to compare the total pre-arcing time and the voltage drop along the fuse-element obtained by simulation and experiment. This model is useful to obtain the inverse prearcing time/current characteristics for industrial fuse-elements of various geometries. Future works will consist in carrying out an extension to the three-dimensional case in order to simulate longer pre-arcing times where heat transfer between fuse-element and the filling material is significant. 


\section{Acknowledgments}

The authors would like to thank both for their help through many discussions and their financial support F. Gentils from Schneider Electric, J.C. Perez-Quesada from Mesa and T. Rambaud and J.L. Gelet from Ferraz Shawmut.

\section{References}

[1] Wright A and Newbery P G 2004 Electric Fuses 3rd Edition IEE Power \& Energy Series 49

[2] Daalder J E and Schreurs E F 1983 Arcing phenomena in high voltage fuses EUT report 83-E-137 Eindhoven

[3] Beaujean D A, Newbery P G and Jayne M G 1995 Modelling fuse elements using a C.A.D. software package Proc. Fifth International Conference on Electrical Fuses and their Applications ICEFA Ilmenau Germany 133-142

[4] Lindmayer M 1999 3D simulation of fusing characteristics including the "M-effect" Proc. Sixth International Conference on Electrical Fuses and their Applications ICEFA Torino Italy 13-20

[5] Leach J G, Newbery P G and Wright A 1973 Analysis of high-rupturing-capacity fuse links prearcing phenomena by a finite difference method Proc. IEE 120(9) 987-993

[6] Wilkins R and Mc Ewan P M 1975 AC short-circuit performance of notched fuse element Proc. IEE 122(3) 289-292

[7] Garrido C and Cidras J 1995 Study of different materials as fuse element Proc. Fifth International Conference on Electrical Fuses and their Applications ICEFA Ilmenau Germany 201-206

[8] Garrido C and Cidras J 1999 Study of fuselinks with different t-I curves using a mathematical model Proc. Sixth International Conference on Electrical Fuses and their Applications ICEFA Torino Italy 21-24

[9] Giurgiu V Oarga G and Purdel S 1999 Analysis of thermal phenomena in high-voltage fuse-links Proc. Sixth International Conference on Electrical Fuses and their Applications ICEFA Torino Italy $65-68$

[10] Meng X Z and Wang J M 1987 The simulation of prearcing characteristics of fuse elements in the finite element method Proc. Third International Conference on Electrical Fuses and their Applications ICEFA Eindhoven Netherlands 24-29

[11] Zhang Juan and Ma Zhiying 1995 Mathematic model of two dimensional temperature field and its application in high-voltage current-limiting fuse-link Power System Technology 19(12)

[12] Kurschner H, Ehrhardt A and Nutsch G 1995 Calculation of prearcing time using the finite element method Proc. Fifth International Conference on Electrical Fuses and their Applications ICEFA Ilmenau Germany 156-161

[13] Fernandez L, Canas C, Llobell J, Curiel J, Aspas J, Ruz F and Cavalle F 1995 A model for pre-arcing behaviour simulation of H.V. full-range fuse-links using the finite element method Proc. Fifth International Conference on Electrical Fuses and their Applications ICEFA Ilmenau Germany 162-168

[14] Canas Penuelas C S, Fernandez L and Gonzalez R 1999 Minimum breaking current obtaining in fuses Proc. Sixth International Conference on Electrical Fuses and their Applications ICEFA Torino Italy 69-74

[15] Jakubiuk K and Aftyka W 2003 Heating of fuse-elements in transient and steady-state Proc. Seventh International Conference on Electrical Fuses and their Applications ICEFA Gdansk Poland 181-187

[16] Kawase Y, Mivatake T and Ito S 2000 Heat analysis of a fuse for semiconductor devices protection using 3-D finite element method IEEE Trans. Magn. 36(4) 1377-1380

[17] Wilkins R 1984 Simulation of fuselink temperature rise tests Proc. Second International Conference on Electrical Fuses and their Applications ICEFA Trondheim Sweden 
[18] Sloot J G J 1987 Analog simulations of the heat flow in a high voltage fuse Proc. Third International Conference on Electrical Fuses and their Applications ICEFA Eindhoven Netherlands

[19] Hoffmann G and Kaltenborn U 2003 Thermal modeling of high voltage H. R. C. fuses and simulation of tripping characteristic Proc. Seventh International Conference on Electrical Fuses and their Applications ICEFA Gdansk Poland 174-180

[20] Rochette D and Clain S 2004 Mathematical model and simulation of gas flow through a porous medium in high breaking capacity fuses Int. J. of Heat and Fluid Flow 25 115-26

[21] Rochette D and Bussière W 2004 Pressure evolution during HBC fuse operation Plasma Sources Sci. Technol. 13(2) 293-302

[22] Bussière W and André $\mathrm{P} 2001$ Evaluation of the composition, the pressure, the thermodynamic properties and the monoatomic spectral lines at fixed volume for a SiO2-Ag plasma in the temperature range 5000-25000 K Journal of Physics D 34 1657-1664

[23] Rochette D, Bussière W and André P 2004 Composition, enthalpy, and vaporization temperature calculation of $\mathrm{Ag}-\mathrm{SiO} 2$ plasmas with air in the temperature range from 1000 to $6000 \mathrm{~K}$ and for pressure included between 1 and 50 bars Plasma Chemistry and Plasma Processing 24(3) $475-492$

[24] JANAF and NIST-JANAF 1998 Thermochemical tables 4th edition, ed M W Chase J. Phys. Chem. Ref. Data (USA)

[25] Touloukian Y S 1970 Thermal conductivity: metallic elements and alloys Vol. 1 Touloukian (Y.S.) series (ed.), Ho (C.Y.) Series technical ed. IFI/Plenum New York Washington

[26] Matula R A 1979 Electrical resistivity of copper, gold, palladium and silver J. Phys. Chem. Ref. Data $8(4)$

[27] Berger A E, Brézis H and Rogers J C W 1979 A numerical method for solving the problem $u_{t}-\Delta f(u)=0$ R.A.I.R.O. Numerical analysis 13(4) 297-312

[28] Masserey A, Rappaz J, Rozsnyo R and Touzani R 2003 Power formulation for the optimal control of an industrial induction heating process for thixoforming International Journal of Applied Electromagnetics and Mechanics 18 1-7

[29] Bussière W, Velleaud G, Gelet J L, Gentils F, Latchimy T, Perez-Quesada J C, Rambaud T and André P A specific test device for HBC fuses. Influence of the closing angle In preparation

[30] Touzani R. Object Finite Element Library, Copyright (C)1998-2006 Rachid Touzani, (http://www.ofeli.net). 